\title{
Interest and Growth
}

\author{
Andrew P. Leung $\mathbb{1}$ \\ Melbourne, Australia \\ Email: apleung8@gmail.com
}

How to cite this paper: Leung, A.P. (2021) Interest and Growth. Journal of Mathematical Finance, 11, 267-293.

https://doi.org/10.4236/jmf.2021.112016

Received: February 8, 2021

Accepted: May 17, 2021

Published: May 20, 2021

Copyright (c) 2021 by author(s) and Scientific Research Publishing Inc. This work is licensed under the Creative Commons Attribution International License (CC BY 4.0).

http://creativecommons.org/licenses/by/4.0/

\begin{abstract}
This paper considers the relationship between population growth and capital accumulation. In general, the relationship is not monotonic; there is a tension between two opposing effects: a higher growth rate diluting capital that has already been accumulated, and the ability of a growing population to generate its own wealth. The strengths of these effects depend on the population structure and the time horizon for each generation to accumulate wealth. This type of analysis has been conducted within a natural Overlapping Generations framework. However, an explicit assumption is needed to allow a comparatively static analysis of levels of population growth and capital accumulation. That assumption is effectively the requirement for each generation to be self supporting in terms of its consumption and contribution to production. We show that this assumption can be justified in theory by a form of economic efficiency with respect to population changes, referred to as demographic efficiency. This analysis is also conducted within the Overlapping Generations model of modern economics. An interesting aspect is the structure of "steady state" economies, that is where each consumer is identical to any other through time. With simple examples and assumptions, this paper demonstrates that a given interest rate can support more than one population growth rate (unlike Samuelson's golden rule, where these rates are equal). The ramifications for non steady state economies are accordingly more complex.
\end{abstract}

\section{Keywords}

Overlapping Generations, Efficiency, Demographic Structure

\section{Introduction}

Since its introduction, the overlapping generations (OLG) model has been considered as the natural framework for explaining savings behavior in relation to a given population. A vast literature has developed with respect to its application. 
The two main streams of the OLG literature have been devoted to the existence of competitive equilibria and the characterization of efficiency.

For the discrete time, finite lifetime model with given endowments, Samuelson found a criterion of efficiency within the OLG framework, referred to as the "golden rule", and discussed it in several papers [1] [2] [3]. Under steady state growth, efficiency is assured if the rate of interest equals the (constant) rate of population growth. This characterization was extended, first with a fundamental result of [4], then by [5]. Most of these efficiency characterizations involve a specific relationship between the interest rate dynamics and that of the population.

In contrast, [6] presents an economy with a (countably) infinite number of infinitely lived agents. As with [7], his commodity space as well as the price space is the space of real sequences. He shows that under standard conditions, if the value of the total endowment of resources is finite, then the first theorem of welfare economics holds, that is, every competitive equilibrium is Pareto optimal. He also shows that this classical result can fail when the value of the total endowments is not finite at equilibrium.

The paper by [8] integrates the approach taken by Balasko and Shell, and Wilson, with that taken by [9] [10] and [11]. The latter approach is from the viewpoint of a general equilibrium economy, where prices reside in the dual of the commodity space. This is the starting point of this paper which, however, is concerned with a different aspect of economies, namely, the role of consumers themselves.

It is also of interest to examine the role of population in achieving efficiency. [12] introduced an OLG model where fertility, via the number of children born, is a decision variable of the parents. It has been claimed [12] [13] that such endogenization guarantees efficiency in OLG models, but questions of convergence in such models have not been given as much prominence as in models assuming exogenous growth.

This paper seeks to extend the efficiency characterizations for OLG models with exogenous population to OLG models with variable population growth. It does this by considering an extended definition of efficiency which embraces variations in population, referred to as demographic efficiency in this paper. It also demonstrates that such an extended concept of efficiency holds for a price equilibrium, if all generations of consumers follow a lifetime budget constraint. This lifetime budget is measured in terms of present values of income and expenditure with discounting at the interest rates supporting the equilibrium.

This lifetime budget constraint and its constraints provide the starting point for studies in capital accumulation and population growth as undertaken recently by [14], Equations (3) and (4), and [15], Equation (7). However, the framework adopted in this paper is less restrictive than that used by the authors quoted. Not only are mortality characteristics permitted to evolve through time, but both interest rates and population growth are endogenous to the model and need not underpin steady states. Given a production technology, the only ex- 
ogenous feature of the model, there are quantitative relationships between the endogenous variables may be used to explore the link between population and capital accumulation, surely a relevant objective in retirement incomes policy.

The lifetime budget constraint is also reminiscent of the "no surplus" condition for efficiency in finite economies [16], under which each generation has an incentive to participate in trade. However, the lifetime budgets introduced in this paper are concerned more with the nature of each generation's endowment, rather than with the relative values of endowments and allocations.

This paper is organized as follows. First, we introduce a general framework, in discrete time, for OLG models, covering consumer demographics, consumption and production. Second, several notions of efficiency are discussed, including one based on endogenous population. Third, we state a sufficient condition for demographic efficiency. Fourth, the major contribution of this paper is in discussing the extent to which that condition is also necessary. The paper concludes with some implications for retirement incomes policy and with some possible extensions.

This paper is organized as follows. First, we introduce a general framework, in discrete time, for OLG models, covering consumer demographics, consumption and production. Second, several notions of efficiency are discussed, including one based on endogenous population. Third we state a sufficient condition for demographic efficiency. Fourth, the major contribution of this paper is in discussing the extent to which that condition is also necessary. The paper concludes with some implications for retirement incomes policy and with some possible extensions.

\section{Framework}

In this paper, we adopt an OLG model with production in the spirit of [17]. The only refinements made to this model are the possibility of life for multiple periods, and a general population structure for consumers, which are detailed as follows.

\subsection{Demographics}

Consumers are distinguished only by time of birth, and the set of such consumers born at a particular time $s$ is referred to as a generation. Thus all members of a generation are assumed to be identical.

The total population of consumers at time $t$ may be described by a population function $\ell(s, t)$, non-zero only for $t \geq s$, representing the number of consumers born at time $s$ who survive to time $t$ [[18], Chapter 18]. The mortality rate at time $t$ of consumers born at time $s$ is thus $q(s, t)=\frac{\ell(s, t)-\ell(s, t+1)}{\ell(s, t)}$. Consumers who die during the period $[t, t+1)$ are assumed to die just before time $t+1$. The function $\hat{\ell}(s)=\sum_{t} \ell$, which is related to each generation's 
expectation of life, provides a measure on generations $s \in \mathbb{Z}$.

The total population of consumers at time $t$ is given by $L(t)=\sum_{s} \ell(s, t)$. For a particular generation $s$, the ratio $\ell(s, t) / \ell(s, s)$ may be regarded as the probability at birth of survival to time $t[[18]$, Chapter 3].

Each generation is supposed to be endowed with labor, described by a given labor supply function $b(s, t)$ which measures the number of working hours available during the period $[t, t+1)$. The aggregate labor supply is thus ${ }^{1}$ $B(t)=\sum b(s, t) \ell(s, t)$.

Remark 1. The above framework relates to a closed economy. Immigration can be allowed for by defining an expanded population function $\ell(s, z, t)$ where $z$ is the age at migration and $\ell(s, z, t)=0$ for $z \geq s$. Emigration then corresponds to $\ell(s, z, t)=0$ for $z \leq s$. However, that is beyond the scope of this paper.

\subsection{Consumption}

At any time, there is a finite number $n$ commodities in the economy. The commodity space at any time is the $n$ dimensional Euclidean space $\mathbb{Z}^{n}$, which is equipped with the Euclidean norm $|\cdot|^{2}$

A state of the economy is described by a consumption function $\mathbf{c}(s, t): \mathbb{Z}^{2} \rightarrow \mathbb{Z}^{n}$, for each member of the generation $s$ at time $t^{3}$ The aggregate consumption at time $t$ is thus $\mathbf{C}(t)=\sum \mathbf{c}(s, t) \ell(s, t)$. A given or initial endowment of consumption is denoted $\mathbf{c}^{*}(s, t) \stackrel{s}{\in} \mathbb{Z}^{n}$. We are concerned mainly with consumption functions that are bounded uniformly across time and generations.

As the measurable population function $\ell(s, t)$ induces a measure on $\mathbb{Z}^{2}$, we thus consider the following Lebesgue spaces in various contexts:

- $l_{\infty}\left(\mathbb{Z}^{2}, \mathbb{Z}^{n}\right)$ : the space of all bounded functions $f: \mathbb{Z}^{2} \rightarrow \mathbb{Z}^{n}$. The norm of this space is taken to be the essential supremum norm, i.e.

$$
\|\mathbf{f}\|_{\infty}=\sup |\mathbf{f}(s, t)|
$$

- $l_{1}\left(\mathbb{Z}^{2}, \mathbb{Z}^{n}\right)$ : the space of all summable functions $g: \mathbb{Z}^{2} \rightarrow \mathbb{Z}^{n}$. The norm of this space is given by the rule:

$$
\|\mathbf{g}\|_{1}=\sum_{s, t}|\mathbf{g}(s, t)| \ell(s, t)<\infty .
$$

Similarly the total population function $L(t)=\sum \ell$ defines a measure on $\mathbb{Z}$ associated with the spaces $l_{\infty}\left(\mathbb{Z}, \mathbb{Z}^{n}\right)$ and $l_{1}\left(\mathbb{Z}, \mathbb{Z}^{n}\right)$, and with their corresponding norms.

where there is no ambiguity, for $m=1,2$ we write $l_{\infty}\left(\mathbb{Z}^{m}\right)$ and $l_{1}\left(\mathbb{Z}^{m}\right)$, or even $l_{\infty}$ and $l_{1}$, for the spaces $l_{\infty}\left(\mathbb{Z}^{2}, \mathbb{Z}^{n}\right)$ and $l_{1}\left(\mathbb{Z}^{m}, \mathbb{Z}^{n}\right)$, respectively.

${ }^{1}$ This will be applied as an input to production.

${ }^{2} \mathrm{We}$ use this notation rather than the more usual $\|\cdot\|$ in order to avoid confusion with the $L_{p}$ norms introduced subsequently.

${ }^{3}$ Note that $\mathrm{n}$-dimensional variables are generally denoted as vectors in bold type. 


\subsection{Consumer Utility}

We assume that the preferences of consumers in a generation $s$ over various consumption paths $\mathbf{c}(s, t)$ are given by a utility summation of the form

$$
U^{s}[\mathbf{c}]=\sum_{t} u[\mathbf{c}(s, t)] \ell(s, t)
$$

where $u(\cdot)$ is the instantaneous utility function at any point of time. Where there is no ambiguity we omit the reference to the generation $s$ and write simply $U[\mathbf{c}]$. This imposes an additively separable structure on each consumer's overall utility, implicit in taking a summation. The utility summation is similar to a Von Neumann-Morgenstern type of expected utility, with discounting via the population parameter $\ell(s, t)$, regarded as a survival probability to time $t$ for the generation $s$.

The instantaneous utility function $u(\mathbf{c})$ is assumed to be concave, monotonic, and twice continuously differentiable. In order to make sense of the utility summations, we need to ensure that only allocations $\mathbf{c}^{*}$ for which $u\left(\mathbf{c}^{*}\right)$ is bounded are considered. We can achieve this by requiring either $u(\cdot)$ to be bounded, with $\bar{u}=\sup u\left(\mathbf{c}^{*}\right)<\infty$, or else $\mathbf{c}^{*}$ itself to be bounded.

In general only utility functions satisfying a particular uniform property for their second derivatives are considered.

Definition 2. The curvature of an instantaneous utility function $u(\mathbf{c})$, at the point $\mathbf{c}$ in the direction of the unit vector $\mathbf{x}$, is defined as the quantity

$$
\kappa(\mathbf{c}, \mathbf{x})=-\frac{\mathbf{x}^{\mathrm{T}} u^{\prime \prime}(\mathbf{c}) \mathbf{x}}{\left|u^{\prime}(\mathbf{c})\right|}
$$

where $u^{\prime \prime}(\mathbf{c})$ is the Hessian matrix corresponding to the function $u(\mathbf{c})$.

$A$ utility function is then said to have bounded minimum (resp. maximum) curvature if there is a uniform lower (resp. upper) bound to $\kappa(\mathbf{c}, \mathbf{x})$ for all $\mathbf{c}, \mathbf{x}$.

This is related to the coefficient of risk aversion introduced in [19]. Further assumptions on the behavior of $u(\mathbf{c})$ will be made in context ${ }^{4}$.

\subsection{Production}

For simplicity, we adopt the usual assumption that production is instantaneous via a net production function $\mathbf{F}(\mathbf{K}, B)$ for capital $\mathbf{K}$ and aggregate labor supply $B$, as introduced in Section 2.1. Under these circumstances, $\mathbf{K}$ is an $n$-dimensional vector of commodities, representing the rates at which inputs are used in the production process, and $\mathbf{F}$ is similarly a vector representing outputs. We also adopt the conventional assumptions that the function $\mathbf{F}(\mathbf{K}, B)$ is concave in its arguments. Other conditions on $\mathbf{F}$ will be made in context.

\section{Prices}

The economic framework introduced above is completely abstract and does not

${ }^{4}$ Since $u$ is a function of a commodity bundle, the gradient $u^{\prime}$ is a vector in $\mathbb{Z}^{n}$. Similarly the second derivative $u^{\prime \prime}$ should be interpreted as a Hessian matrix. 
involve the existence of supporting prices. The concept of an "efficient" allocation may be defined under this abstract framework, as set out in the next section.

In practice, abstract economies are rarely of interest for their own sake, but only to the extent that they are supported by prices. To introduce the role of prices, we adopt the following notation.

Let $\mathbf{p}(t)$ be the price vector of commodities, $r(t)$ be the instantaneous rate of interest and $\mathbf{w}(t)$ the wage rate at time $t$. Further, let $T^{s}$ denote the present value of net transfers to generation $s$ from other generations per head of population.

Then the value of labor income is $\ell b \mathbf{p} \cdot \mathbf{w}$ at time $t$. Taking discount factors

$$
\gamma(t)=\exp \left[-\sum_{0}^{t} r(z)\right]
$$

the present value of labor and other endowments, allowing for interest as well as the probability of survival for generation $s$, is

$$
\begin{aligned}
& \sum_{s}^{\infty} \frac{\gamma(z)}{\gamma(s)} \ell(s, z) \mathbf{p}(z) \cdot \mathbf{c}(s, z) \\
& =\sum_{s}^{\infty} \frac{\gamma(z)}{\gamma(s)} \ell(s, z) b(s, z) \mathbf{p}(z) \cdot \mathbf{w}(z)+\ell(s, s) T^{s} .
\end{aligned}
$$

Writing $\mathbf{v}(t)=\gamma(t) \mathbf{p}(t)$ as the (discounted) prices, we can define (aggregate) income for generation $s$ by

$$
Y^{s} \equiv \sum_{s}^{\infty} \ell(s, z) b(s, z) \mathbf{v}(z) \cdot \mathbf{w}(z)+\gamma(s) \ell(s, s) T^{s} .
$$

Thus the lifetime value of labor for generation $s$ can be expressed by summations such as $\sum \ell(s, t) b(s, t) \mathbf{v}(t) \cdot \mathbf{w}(t)$, whereas the value of lifetime consumption can be expressed by summations such as $\sum_{t} \ell(s, z) \mathbf{v}(t) \cdot \mathbf{c}(s, t)$ for $\mathbf{c}(s, t) \in l_{\infty}\left(\mathbb{Z}^{2}\right)$.

It may be noticed in passing that the lifetime present values appearing above allow for discounting both for interest (via $\mathbf{v}(t)$ ) as well as the probability of survival (via $\ell(s, t)$ ). This is based in effect on aggregating over all members of each generation. An alternative derivation is to apply the redistribution mechanism suggested by [20]. Under that mechanism, redistribution of wealth is effected individually, each member of a generation receiving an annuity whilst alive, in return for surrender of wealth on death. This individual wealth accumulation relation is identical to the aggregate relation above, which is not surprising as the principles underlying actuarial equivalence of annuity prices and the redistribution mechanism are identical.

The value of aggregate future consumption is thus $\sum_{t>0} \mathbf{v}(t) \cdot \mathbf{C}(t)$. If this value is finite, the discounted prices $\mathbf{v}(t)$ may be regarded as elements of the dual space $l_{1}(\mathbb{Z})$, in which case we refer to $\mathbf{v}(t)$ as a discount function.

\subsection{Topologies of Consumption and Price Spaces}

Under the weak topology for $l_{\infty}$, the pair $\left\langle l_{\infty}, l_{1}\right\rangle$ is a dual pair or dual sys- 
tem [21]. For $m=1,2$ the strong dual space of each $l_{\infty}\left(\mathbb{Z}^{m}, \mathbb{Z}^{n}\right)$, denoted $l_{\infty}\left(\mathbb{Z}^{m}, \mathbb{Z}^{n}\right)^{\prime}$, is isometric to $b a\left(\mathbb{Z}^{m}, \mathbb{Z}^{n}\right)$, the space of bounded additive $\mathbb{Z}^{n}$ valued set functions on $\mathbb{Z}^{m}$, and includes $l_{1}\left(\mathbb{Z}^{m}, \mathbb{Z}^{n}\right)$ as a proper subspace [[22], §IV.8.16].

Where there is no ambiguity, we similarly write $b a\left(\mathbb{Z}^{m}\right)$ or even just $b a$. Besides the strong topologies induced by the norm in each space, this paper considers the weak topologies ${ }^{5} \sigma\left[b a, l_{\infty}\right]$ on $b a$ and $\sigma\left[l_{1}, l_{\infty}\right]$ on $l_{1}$. Under the natural embedding of $l_{1}$ in $b a$ and with the weak topology $\sigma\left[b a, l_{\infty}\right]$, Goldstine's theorem states that $l_{1}$ is a dense subset of ba [[22], §V.4.5].

Finally, in order to apply results in the literature, we consider the Mackey topology $\tau\left(l_{\infty}, l_{1}\right)$, defined as the finest topology of the dual pair $\left\langle l_{\infty}, l_{1}\right\rangle,[[21]$, \$6.7]. This is the topology of uniform convergence on all convex compact subsets of $l_{1}$ in the $\sigma\left[l_{1}, l_{\infty}\right]$ topology. Following a characterization due to [23], convergence in the Mackey topology for $l_{\infty}$ may be shown to be equivalent to that in the so-called strict topology, which is based on the semi-norms

$$
\beta(\mathbf{c})=\sup |\beta(t) \cdot \mathbf{c}(t)|, \mathbf{c}(t) \in l_{\infty}
$$

for any function $\beta(t) \rightarrow 0$.

\subsection{Utility Maximization}

In an economy with prices, it is necessary to specify how consumers choose consumption paths. This follows the familiar maximization of utility subject to the lifetime budget constraint above. That is, each generation's problem is:

$$
\max _{c} U^{s}[\mathbf{c}]=\sum_{t} u(\mathbf{c}) \ell \text { such that } Y^{s}=\sum_{t} \mathbf{v} \cdot \mathbf{c} \ell \text {. }
$$

Each consumer's problem is analogous to that in a finite economy, where the commodities are distinguished not only by type but also by time of consumption. The value of lifetime income is then given by the variable $Y^{s}$ defined above.

To solve this problem it is possible to apply standard optimization techniques. An interior solution is guaranteed by strict concavity of $u(\mathbf{c})$. The first order condition for an interior solution $\mathbf{c}=\mathbf{c}^{*}$ is $u^{\prime}\left(\mathbf{c}^{*}\right)=\mathbf{c}^{*}(s, t) \mathbf{v}(t)$ for all $s$ and $t$. The extent to which such a condition is also necessary is considered in Proposition 15 of this paper. Since $Y^{s}=\sum \mathbf{v} \cdot \mathbf{c} \ell$ is the value of future consumption, or equivalently the value of income, then the factors $\lambda(s)$ are just the marginal (indirect) utilities with respect to income for each generation.

\section{Economic Efficiency}

A price equilibrium may be defined as an allocation $\mathbf{c}^{*}$ and discounted prices $\mathbf{v}$ where consumers maximize utility as follows.

Definition 3. Given the demographic structure $\ell(s, t)$, a price equilibrium $\left\{\mathbf{c}^{*}, \mathbf{v}\right\}$ is defined as an allocation $\mathbf{c}^{*}(s, t)$ together with a set of prices (or dis-

\footnotetext{
${ }^{5}$ Sometimes referred to as the weak ${ }^{\star}$ topology.
} 
count factors) $\mathbf{v}(t)$ such that each generation maximizes utility under a lifetime budget constraint, that is $\mathbf{c}=\mathbf{c}^{*}$ solves a utility maximization problem of the form set out in Equation (2).

To examine efficiency of an economy, we need to consider the effect of varying the allocation $\mathbf{c}^{*}(s, t)$ to some other allocation $\mathbf{c}(s, t)$. In general we are interested only in prospective reallocations, that is where allocations are varied after some fixed time. Without loss of generality we may take this time to be $t=0$. Thus $\mathbf{c}(s, t)=\mathbf{c}^{*}(s, t)$ for $t<0$.

Definition 4. An improving allocation $\mathbf{c}(s, t)$ for the given allocation $\mathbf{c}^{*}(s, t)$ is one for which $U^{s}(\mathbf{c}) \geq U^{s}\left(\mathbf{c}^{*}\right)$, with strict inequality for at least one generation $s$.

The allocation $\mathbf{c}^{*}(s, t)$ is then efficient with respect to a given class if no improving allocation exists for $t \geq 0$ from among a class of feasible allocations.

As consumption paths extend over a continuum, and there is a continuum of consumers, the traditional welfare theorems of finite economies need not hold. In general, additional conditions are needed to ensure both the existence of efficient allocations and their implementation as price equilibria.

The above definition of efficiency rests upon the class of feasible allocations that are to be admitted. We consider three such classes below, which result in successively stronger concepts of efficiency.

Definition 5. An allocation is (allocatively) efficient if there are no improving allocations which preserve aggregate consumption.

The feasible allocations $\mathbf{c}(s, t)$ are therefore those which maintain aggregate consumption for a given initial allocation $\mathbf{c}^{*}(s, t)$, that is

$$
\mathbf{C}(t)=\sum_{s} \mathbf{c}(s, t) \ell(s, t)=\mathbf{C}^{*}(t)=\sum_{s} \mathbf{c}^{*}(s, t) \ell(s, t) .
$$

This is a concept of efficiency applicable to a pure exchange economy, that is, one in which the aggregate level of consumed commodities is taken as fixed, without regard to their source.

Definition 6. The allocation $\mathbf{c}^{*}(s, t)$ is efficient in production if no improving allocation exists for $t \geq 0$ which are compatible with the production technology.

The feasible allocations in this case are those allocations $\mathbf{c}(s, t)$ satisfying the condition:

$$
\mathbf{C}(t)+\mathbf{K}(t+1)=\mathbf{F}(\mathbf{K}, B)+\mathbf{K}(t),
$$

for some capital path $\mathbf{K}(t) \geq 0$.

Remark 7. The function $\mathbf{K}(t)$ may be considered net of capital depreciation.

\section{Variations in Population and Production}

With the production process described in the previous section, it becomes possible to consider the full implications of a change in population. Consider a given population density $\ell(s, t)$, referred to as the planned population, and a hypo- 
thetical variation in future birth rates resulting in a change in the population density $\ell(s, t)$ of the form ${ }^{6} \Delta \ell=n(s) \ell$, where $n(s)$ is non-zero only for $s \geq 0$. For a given capital path $K(t)$, this will allow production to vary through its dependence on aggregate labor $B(t)$, and hence consumption $\mathbf{c}(s, t)$ may also vary. Let us now consider how efficiency is affected by such variation.

If $\Delta \ell$ and $\Delta \mathbf{c}$ denote the changes in population and consumption, the corresponding changes in aggregate consumption $\mathbf{C}$ and aggregate labor supply $B(t)$ are given by:

$$
\begin{aligned}
\Delta B & =\sum_{s} b(s, t) n(s) \ell(s, t) \\
\Delta \mathbf{C} & =\sum_{s} \Delta(\mathbf{c} \ell)=\sum_{s} \mathbf{c} n(s) \ell(s, t)+\sum_{s} \ell \Delta \mathbf{c}+\sum_{s} n \ell \Delta \mathbf{c} .
\end{aligned}
$$

Notice that the variation $n \ell \Delta \mathbf{c}$ is of the second order.

A classical instantaneous production function $\mathbf{F}(\mathbf{K}, B)$ must also satisfy the constraint 3 , so that

$$
\Delta \mathbf{C}=\Delta \mathbf{F} .
$$

Definition 8. An allocation is demographically efficient if there are no improving allocations that may be paired with a variation of future birth rates in such a way that the corresponding variations $\{\Delta \mathbf{c}, n\}$ satisfy(3) and (4) above.

This is the last (and the strongest) case of the general efficiency notion introduced in Section 4. It may be noted that, where capital is not varied, concavity of the production function implies the following relationship:

$$
\Delta \mathbf{C}=\Delta \mathbf{F} \leq \mathbf{F}_{B} \Delta B=\mathbf{w}(t) \Delta B .
$$

where $\mathbf{w}(t)=\mathbf{F}_{B}$ is the wage rate.

\section{Sufficient Conditions}

We state without proof sufficient conditions for sufficiency in each of the senses defined above. They are straightforward to demonstrate by repeated applications of Fubini's theorem, which is stated and proved in [24].

Proposition 9 (Demographic Efficiency). Suppose that

- $\left\{\mathbf{c}^{*}, \mathbf{v}\right\}$ is a price equilibrium;

- the utility is bounded and has bounded minimum curvature,

- the marginal condition $u^{\prime}\left(\mathbf{c}^{*}\right)=\lambda(s) \mathbf{v}$ holds, and

- $\mathbf{v}(t) \in L_{1}(\mathbb{Z})$ is summable.

If, in addition,

- total consumption has finite value $\sum_{t} \mathbf{v} \cdot \mathbf{C}^{*}<\infty$.

- for all generations $s$, we also have

$$
\sum_{t} \mathbf{v} \cdot \mathbf{F}_{B} b \ell=\sum_{t} \mathbf{v} \cdot \mathbf{c}^{*} \ell
$$

then the allocation $\mathbf{c}^{*}(s, t)$ is demographically efficient.

${ }^{6}$ It is implicitly assumed that any additional members of a particular generation must be identical to its original members, which is a matter of equity. 
Note that the condition $\sum_{t} \mathbf{v} \cdot \mathbf{C}^{*}<\infty$ is automatically satisfied if the average allocation $\frac{\mathbf{C}(t)}{L(t)}$ is bounded and $\mathbf{v}(t) \in l_{1}(\mathbb{Z})$.

The interesting condition for demographic efficiency contained in Proposition 9 is that, for almost all generations, transfers are zero. That is, a form of lifetime budget constraint providing that the present value of future consumption must equate at birth with the present value of future labor income, with present values allowing for discounting for both interest (via the factors $\mathbf{v}$ ) and probability of survival (via the factors $\ell$ ). This condition does not involve bequests to or from other generations, or contributions from firms' profits. In fact the condition is a special case of the first-order condition set out in Section 3, with zero net transfers $T^{s}=0$ for each generation.

It is also of interest to consider conditions for ensuring efficiency in production. The following result mirrors the condition for finite economies:

Proposition 10 (Productive Efficiency). Under the conditions of Proposition 9, suppose that $\mathbf{v}(t) \cdot \mathbf{F}_{\mathbf{K}}=-[\mathbf{v}(t)-\mathbf{v}(t-1)]$ for the capital path $\mathbf{K}(t)$ satisfying Equation (4). Then the allocation $\mathbf{c}^{*}$ is efficient in production.

Whilst variations in future aggregate population, consumption, production and capital can all occur together, it is convenient to assume that capital is not varied, at least for the purposes of examining necessary conditions for demographic efficiency.

\section{Necessary Conditions}

It is important to consider whether the sufficiency conditions presented in the previous section are the best possible.

\subsection{Allocative Efficiency}

\subsubsection{Existence of a Separating Functional}

Suppose that $\mathbf{c}^{*}(s, t) \in l_{\infty}\left(\mathbb{Z}^{2}\right)$ is an efficient allocation. In direct analogy with discrete economies we wish to consider whether a separating functional in the dual space $b a(\mathbb{Z})$ exists, that is one which separates the allocation from improving allocations. Although it is shown in this section that a separating functional exists under certain technical conditions, such separating functionals are not referred to as prices. As the sufficiency results from the previous section suggest that prices should possess integrability properties, we reserve the term price for a functional in the subspace $l_{1}(\mathbb{Z}) \subset b a(\mathbb{Z})$.

We first set out a lemma which introduces the consumption averaging operator $\pi$.

Lemma 11 (An Open Mapping). Let the map

$$
\pi: l_{\infty}\left(\mathbb{Z}^{2}\right) \rightarrow l_{\infty}(\mathbb{Z})
$$

be defined by taking average consumption over generations at a particular time, in the following way 


$$
\pi \mathbf{c}=\frac{\sum_{s} \mathbf{c} \ell}{\sum_{s} \ell}=\frac{\mathbf{C}(t)}{L(t)}
$$

for a bounded consumption function $\mathbf{c} \in l_{\infty}\left(\mathbb{Z}^{2}\right)$. Then $\pi$ is an open map (that is, maps open sets to open sets).

The above lemma allows us to apply a separation theorem for open sets to obtain the following general result.

Proposition 12. Suppose that:

- $\mathbf{c}^{*}(s, t) \in L_{\infty}\left(\mathbb{Z}^{2}\right)$ is a bounded efficient allocation;

- the utility function $u(\cdot)$ has bounded maximum curvature;

- the magnitude of its gradient $\left|u^{\prime}(\cdot)\right|$ is bounded above.

Then:

- the set of improving allocations $\mathcal{P}\left[\mathbf{c}^{*}\right]=\left\{\mathbf{c} \in l_{\infty}\left(\mathbb{Z}^{2}\right)\right.$ improves $\left.\mathbf{c}^{*}\right\}$ has an interior point, and

- there exists a continuous functional $\mathbf{v} \in b a(\mathbb{Z})$ such that $\langle\mathbf{v}, \pi \Delta \mathbf{c}\rangle \geq 0$ for any improving variation $\Delta \mathbf{c}(s, t) \in l_{\infty}\left(\mathbb{Z}^{2}\right)$.

The above Proposition has several consequences, one of which is stated without proof as follows.

Corollary 13. Under the conditions of Proposition 12, the continuous functional $\mathbf{v} \in b a(\mathbb{Z})$ for any improving variation $\Delta \mathbf{c}(s, t) \in l_{\infty}\left(\mathbb{Z}^{2}\right)$ has the property that $\langle\mathbf{v}, \Delta \mathbf{c}\rangle \geq 0$ for all generations $s$.

This allows us to confirm that the separating functional demonstrated in Proposition 12 does indeed separate improving allocations.

Lemma 14. Under the same conditions as assumed for Proposition 12, suppose the separating functional is represented as a discount function $\mathbf{v}(t) \in l_{1}(\mathbb{Z})$. Then it is unique up to a multiplicative constant, and we have a decomposition $u^{\prime}\left(\mathbf{c}^{*}\right)=\lambda(s) \mathbf{v}(t)$ for some scalar function $\lambda(s)$.

The above results will prove to be useful in deriving conditions under which the supporting functional is a discount function.

\subsubsection{Existence of a Discount Function}

While Proposition 12 ensures the existence of a separating functional for efficient allocations, it is very natural to ask whether conditions exist to guarantee that it is a discount function. The following result, based on the characterization of purely finitely additive measures of Yoshida-Hewitt, provides a partial answer to this question.

Proposition 15 (Existence of a Discount Function). Under the same conditions as assumed for Proposition 12, suppose $\mathbf{c}^{*}(s, t) \in l_{\infty}\left(\mathbb{Z}^{2}\right)$ is an efficient allocation and the separating functional $\mathbf{v} \in b a(\mathbb{Z})$ has the property that $\mathbf{v}\left(\mathbf{c}^{*}\right)$ is non-zero for some generation $s$. Then the countably additive component of $\mathbf{v}(t) \in l_{1}(\mathbb{Z})$, denoted $\mathbf{v}_{c}(t)$, is also a separating functional, and satisfies the relation $u^{\prime}\left(\mathbf{c}^{*}\right)=\lambda(s) \mathbf{v}(t)$ for some scalar function $\lambda(s)$.

The first implication of the above Proposition provides for the existence of a discount price structure $\mathbf{v}(t)$. The decomposition $u^{\prime}\left(\mathbf{c}^{*}\right)=\lambda(s) \mathbf{v}(t)$ is the 
continuous time analog of the first order condition applying in discrete economies. This decomposition is consistent with the conditions holding for price equilibria described in Section 5. In fact it implies that the allocation $\mathbf{c}^{*}$ is a price equilibrium for the supporting prices given by $\mathbf{v}(t)$ and that these prices are unique, up to a multiplicative constant. Thus the Second Welfare Theorem holds for the OLG models of this paper under the assumptions made.

\subsection{Demographic Efficiency}

The sufficient condition for demographic efficiency, set out in Proposition 9, represents a form of lifetime budget constraint for individual generations without any reference to bequests or profit contributions from firms' production. It is a more difficult matter to show that this condition is also, under certain reasonable conditions, a necessary one. As the proofs are intricate and very technical, they are relegated to the Appendix.

If $\left\{\mathbf{c}^{*}, \mathbf{v}\right\}$ is a demographically efficient allocation, then it is also allocatively efficient, and hence under the conditions of Proposition 15 a separating discount function $\mathbf{v}(t) \in l_{1}(\mathbb{Z})$ exists. Consider a variation in population structure $\{\Delta \mathbf{c}, n\}$ of the form set out in Definition 8, where $\Delta \ell=n(s) \ell$ for some bounded function $n \in l_{\infty}(\mathbb{Z})$. This induces a change in aggregate labor $\Delta B=\sum b(s, t) n(s) \ell$ and a change in production $\Delta \mathbf{F}=\mathbf{F}(\mathbf{K}, B+\Delta B)-\mathbf{F}(\mathbf{K}, B)$.

The feasible consumption functions $\mathbf{c} \in l_{\infty}\left(\mathbb{Z}^{2}\right)$ which are compatible with the varied production must satisfy the physical constraint

$$
\sum_{s} \Delta \mathbf{c} \ell+\sum_{s} \mathbf{c}^{*} n \ell=\Delta \mathbf{C} \leq \Delta \mathbf{F} .
$$

Notice that the second order term $\sum n \ell \Delta \mathbf{c}$ appearing in Equation (4) has been omitted for simplicity. This has the physical interpretation that the variation in population $n(s) \ell$ is provided with the original consumption $\mathbf{c}^{*}$ and not the varied consumption c. This is correct up to the second order for small variations in population; however the issue is dealt with in greater detail below.

The set of demographically feasible allocations may thus be defined as those which are compatible with the change in population and production, denoted by

$$
\mathcal{Q}\left[\mathbf{c}^{*}\right]=\left\{\mathbf{c} \in l_{\infty}\left(\mathbb{Z}^{2}\right) \mid \sum_{s}\left(\mathbf{c}-\mathbf{c}^{*}\right) \ell+\sum_{s} \mathbf{c}^{*} n \ell \leq \Delta \mathbf{F} \text { for some } n \in l_{\infty}(\mathbb{Z})\right\} .
$$

It should be noted in this definition that an increase in births with $n(t)>0$ leads to aggregate consumption being increased, all else being equal, as the result of a larger population. However, this can be offset by consumption per head changing from $\mathbf{c}^{*}(s, t)$ to $\mathbf{c}(s, t)$.

It should also be noted that the set $\mathcal{Q}\left[\mathbf{c}^{*}\right]$ is much larger than the set of feasible allocations for allocative efficiency, which comprise only those allocations $\mathbf{c} \in l_{\infty}\left(\mathbb{Z}^{2}\right)$ such that $\pi \mathbf{c} \leq \pi \mathbf{c}^{*}$. Hence a discount function for allocative efficiency in the sense of Proposition 15 is not necessarily a separating functional for demographic efficiency. 
The main result of this paper is the following.

Proposition 16 (Necessary Condition for Demographic Efficiency) Suppose that $\mathbf{c}^{*}$ is a demographically efficient price equilibrium under the conditions of Proposition 15 with discount function $\mathbf{v}(t) \in l_{1}(\mathbb{Z})$. Suppose also that.

- the lifetime of each generation is finite, so that $\ell(s, t)$ has finite support for each $s$.

- the production function has bounded second derivatives $\left|\mathbf{F}_{B B}\right| \leq M$, say.

Then the discount function $\mathbf{v}(t)$ separates demographically feasible allocations $\mathcal{Q}\left[\mathbf{c}^{*}\right]$ from improving allocations $\mathcal{P}\left[\mathbf{c}^{*}\right]$. Further, for all generations $s$, we have the lifetime budget constraint

$$
\sum_{t} \mathbf{v} \cdot \mathbf{F}_{B} b \ell d t=\sum_{t} \mathbf{v} \cdot \mathbf{c}^{*} \ell .
$$

Remark 17. It may be noticed that the definition of feasible allocations actually allocates the initial consumption plan $\mathbf{c}^{*}$ to the new born $n \ell$ and not the revised consumption c. An equitable allocation would result in the feasible allocations being defined as:

$$
\hat{\mathcal{Q}}\left[\mathbf{c}^{*}\right]=\left\{\mathbf{c} \in l_{\infty}\left(\mathbb{Z}^{2}\right) \mid \sum_{s}\left(\mathbf{c}-\mathbf{c}^{*}\right) \ell+\sum_{s} \mathbf{c} n \ell \leq \Delta \mathbf{F} \text { for some } n \in l_{\infty}(\mathbb{Z})\right\}
$$

which unfortunately is not a convex set. This technical difficulty may be overcome as follows.

Suppose $\mathbf{c}=\mathbf{c}^{*}+\Delta \mathbf{c} \in l_{\infty}\left(\mathbb{Z}^{2}\right)$ is a feasible allocation in $\mathcal{Q}\left[\mathbf{c}^{*}\right]$ for some population variation $n \in l_{\infty}(\mathbb{Z})$ with $\|\Delta \mathbf{c}\|_{\infty},\|n\|_{\infty}<1$. Then the variations $\{\varepsilon \Delta \mathbf{c}, \varepsilon n\}$ also establish an equitable allocation in $\hat{\mathcal{Q}}\left[\mathbf{c}^{*}\right]$ for sufficiently small $\varepsilon>0$ since

$$
\begin{aligned}
& \varepsilon \sum_{s} \Delta \mathbf{c} \ell+\varepsilon \sum_{s} \mathbf{c}^{*} n \ell+\sum_{s} \varepsilon^{2} \Delta \mathbf{c} n \ell \\
& <\varepsilon[\mathbf{F}(\mathbf{K}, B+\Delta B)-\mathbf{F}(\mathbf{K}, B)]+\sum_{s} \varepsilon^{2} \Delta \mathbf{c} n \ell \\
& <\varepsilon \mathbf{F}_{B} \sum_{s} b n \ell+\sum_{s} \varepsilon^{2} \Delta \mathbf{c} n \ell \\
& <\mathbf{F}(\mathbf{K}, B+\varepsilon \Delta B)-\mathbf{F}(\mathbf{K}, B)+\frac{1}{2} \varepsilon^{2} M \Delta B+\varepsilon^{2} \sum_{s} \Delta \mathbf{c} n \ell
\end{aligned}
$$

As $\|\Delta \mathbf{c}\|_{\infty},\|n\|_{\infty}<1$ are bounded, there exists $\varepsilon(t)>0$ independent of $\Delta \mathbf{c}$ and $n$ such that

$$
\varepsilon \sum_{s} \Delta \mathbf{c} \ell+\varepsilon \sum_{s} \mathbf{c}^{*} n \ell+\sum_{s} \varepsilon^{2} \Delta \mathbf{c} n \ell \leq \mathbf{F}(\mathbf{K}, B+\varepsilon \Delta B)-\mathbf{F}(\mathbf{K}, B)
$$

so that $\{\varepsilon \Delta \mathbf{c}, \varepsilon n\}$ is in $\hat{\mathcal{Q}}\left[\mathbf{c}^{*}\right]$. Thus define

$$
\begin{aligned}
\mathcal{R}\left[\mathbf{c}^{*}\right]= & \left\{\mathbf{c} \in l_{\infty}\left(\mathbb{Z}^{2}\right) \mid \sum_{s}\left(\mathbf{c}-\mathbf{c}^{*}\right) \ell+\sum_{s} \mathbf{c}^{*} n \ell<\Delta \mathbf{F}\right. \\
& \text { for some } \left.n \in l_{\infty}(\mathbb{Z}) \text { with }\left|\Delta \mathbf{c}_{\infty}\right|,\left|n_{\infty}\right|<\varepsilon(t)\right\}
\end{aligned}
$$

so that $\mathcal{R}\left[\mathbf{c}^{*}\right] \subset \hat{\mathcal{Q}}\left[\mathbf{c}^{*}\right]$. It is easily shown that $\mathcal{R}\left[\mathbf{c}^{*}\right]$ has all the properties of $\mathcal{Q}\left[\mathbf{c}^{*}\right]$, including convexity, that are required to make the proof of Proposition 
16 work. Hence the result holds even for equitable allocations.

Proposition 16 establishes a very similar condition to that for Proposition 9, both being of the form of an individual generation's lifetime budget constraint. The main aspect of interest in this condition is the absence of any bequest involving other generations.

Remark 18. The condition that each generation has a finite lifetime is mild, yet realistic. However, it is not assumed that all lifetimes are equal, or even uniformly bounded. It is needed to allow for the situation that $\|n\|_{\infty}<1$. However infinite individual lifetimes may be accommodated in a continuous time framework.

Remark 19. The proposition does not presume that any of the variables relating to population, interest rates, labor or consumption are either exogenous or endogenous. It simply finds the relationships between these variables if (demographic) efficiency were to hold. Of course, if enough of these variables are exogenous, the remaining ones are endogenous under the proposition. A simple physical analogy is in the position and velocity of a particle at a given time.

\section{Steady State Economies}

\subsection{Discrete Time}

We analyze the relationship between interest rates and growth rates of the economy are discrete time, assuming a steady state economy (i.e. where each consumer is identical to any other). In the next section, we do the same in continuous time, and the results are similar. However, Samuelson's "golden rule" (where interest and growth rates are equal) holds only approximately in discrete time, but precisely in at least one case in continuous time.

\subsection{Aggregates}

Assume the population at time suffers a mortality rate $\mu$, and that those born at time $s$ surviving to time $t$ is $\left[1-\mathrm{e}^{-(\mu+\theta)}\right] \mathrm{e}^{\theta s} \mathrm{e}^{-\mu(t-s)}$. The individual consumption function is assumed to be linear $c(z)=\gamma+r z$. (see below). The total population is using the age $z=t-s$

$$
\begin{aligned}
L(t) & =\left[1-\mathrm{e}^{-(\mu+\theta)}\right] \sum_{s \leq t} \mathrm{e}^{\theta s} \mathrm{e}^{-\mu(t-s)} \\
& =\left[1-\mathrm{e}^{-(\mu+\theta)}\right] \sum_{z \geq 0} \mathrm{e}^{\theta s} \mathrm{e}^{-\mu z} \\
& =\left[1-\mathrm{e}^{-(\mu+\theta)}\right] \mathrm{e}^{\theta t} \sum_{z \geq 0} \mathrm{e}^{-(\mu+\theta) z} \\
& =\mathrm{e}^{\theta t}
\end{aligned}
$$

and similarly total consumption at time $t$ is

$$
\begin{aligned}
C(t) & =\left[1-\mathrm{e}^{-(\mu+\theta)}\right] \sum_{s \leq t} \mathrm{e}^{\theta s} \mathrm{e}^{-\mu(t-s)} c(t-s) \\
& =\left[1-\mathrm{e}^{-(\mu+\theta)}\right] \mathrm{e}^{\theta t} \sum_{z \geq 0} \mathrm{e}^{-(\mu+\theta) z} c(z)
\end{aligned}
$$

and the total labor income (for a unit wage) is 


$$
\begin{aligned}
B(t) & =\left[1-\mathrm{e}^{-(\mu+\theta)}\right] \sum_{s \leq t} \mathrm{e}^{\theta s} \mathrm{e}^{-\mu(t-s)} b(t-s) \\
& =\left[1-\mathrm{e}^{-(\mu+\theta)}\right] \mathrm{e}^{\theta t} \sum_{z \geq 0} \mathrm{e}^{-(\mu+\theta) z} b(z)
\end{aligned}
$$

\subsection{Per Capita Values}

For generation $s$ the value of future consumption at a discount rate $r$ is

$$
\begin{aligned}
& {\left[1-\mathrm{e}^{-(\mu+\theta)}\right] \sum_{t \geq s} \mathrm{e}^{-\mu(t-s)} \mathrm{e}^{-r(t-s)} c(t-s)} \\
& =\left[1-\mathrm{e}^{-(1+\theta)}\right] \sum_{z \geq 0} \mathrm{e}^{-(r+\mu) z} c(z)
\end{aligned}
$$

and the value of future income is similarly

$$
\left[1-\mathrm{e}^{-(1+\theta)}\right] \sum_{z \geq 0} \mathrm{e}^{-(r+\mu) z} b(z)
$$

\subsection{Production}

Capital is assumed to be of the form $F(K, B)=B f(k)$ where capital per labor unit $k=K / B$ is constant, showing constant returns to scale. For a steady state the production function must be of the form:

$$
F(K, B)=B f(k)
$$

where $k=K / B$ is constant and represents the capital/labor ratio.

Thus the steadystate interest rate and wage rate are then:

$$
\begin{gathered}
r=F_{K}=f^{\prime}(k) \\
w=F_{B}=f-\frac{\partial k}{\partial B} f^{\prime}(k)=f-\frac{K}{B} f^{\prime}(k)=f-k r .
\end{gathered}
$$

\subsection{Utility}

We need to justify a steady state consumption path of the form

$$
c=\gamma+r z
$$

for $z=t-s$. To find such a consumption path, the utility function $u(c)$ must satisfy $u^{\prime}(c)=\lambda \mathrm{e}^{-r z}$ so that

$$
u^{\prime}(c)=\frac{\mathrm{d} u}{\mathrm{~d} z} \frac{\mathrm{d} z}{\mathrm{~d} c}=\frac{1}{r} \frac{\mathrm{d} u}{\mathrm{~d} c}=\lambda \mathrm{e}^{-r z}
$$

so that

$$
\frac{\mathrm{d} u}{\mathrm{~d} c}=r \lambda \mathrm{e}^{-r z}=r \lambda \mathrm{e}^{\gamma} \mathrm{e}^{-c}>0
$$

or

$$
u(c)=U-r \lambda \mathrm{e}^{\gamma} \mathrm{e}^{-c}
$$

for some constant $U>0$. We must then have $\frac{\mathrm{d}^{2} u}{\mathrm{~d} c^{2}}<0$, that is diminishing utility to scale. 


\subsection{Example}

We assume the individual rate of consumption function is linear with $c(z)=\gamma+r z$ for an individual of age $z$ (assuming they survive to that age). The consumer utility which justifies this assumption is derived in the previous section.

We also a Cobb-Douglas production function $f=k^{\alpha}$, with $\alpha+\beta=1$ and labor as $b(z)=b$ for $m \leq z \leq n$. The parameter $m>0$ denotes the age of maturity (or productiveness) of an individual, and $n$ that of retirement.

As a numerical example, let $\mu=0.01, r=0.05, \alpha=0.4, m=15, n=40$ and $\gamma=0.1$. Since $r=f^{\prime}(k)=\alpha k^{\alpha-1}=\alpha k^{-\beta}$, then $k=\left(\frac{\alpha}{r}\right)^{1 / \beta}=32$ and $w=\beta k^{\alpha}=2.4$.

\subsection{Equilibrium Conditions}

The conditions for an equilibrium are thus the equality of lifetime consumption and income at a wage rate $w$ for each consumer:

$$
\sum_{z \geq 0} \mathrm{e}^{-(r+\mu) z} c(z)=w \sum_{z \geq 0} \mathrm{e}^{-(r+\mu) z} b(z)
$$

The economy wide production constraint, that it is consumed or employed to increase capital, becomes

$$
\begin{aligned}
F(K, B)= & B f(k)=C+\Delta K \\
= & C+k \Delta B \\
= & f(k)\left[1-\mathrm{e}^{-(\mu+\theta)}\right] \mathrm{e}^{\theta t} \sum_{z \geq 0} \mathrm{e}^{-(\mu+\theta) z} b(z) \\
= & {\left[1-\mathrm{e}^{-(\mu+\theta)}\right] \mathrm{e}^{\theta t} \sum_{z \geq 0} \mathrm{e}^{-(\mu+\theta) z} c(z) } \\
& +k\left[1-\mathrm{e}^{-(\mu+\theta)}\right] \mathrm{e}^{\theta t}\left[1-\mathrm{e}^{-\theta}\right] \sum_{z \geq 0} \mathrm{e}^{-(\mu+\theta) z} b(z)
\end{aligned}
$$

because

$$
\begin{gathered}
B(t)=\left[1-\mathrm{e}^{-(\mu+\theta)}\right] \mathrm{e}^{\theta t} \sum_{z \geq 0} \mathrm{e}^{-(\mu+\theta) z} b(z) \\
\sum_{z \geq 0} \mathrm{e}^{-(\mu+\theta) z} c(z)=\left[f-k\left(1-\mathrm{e}^{-\theta}\right)\right] \sum_{z \geq 0} \mathrm{e}^{-(\mu+\theta) z} b(z) .
\end{gathered}
$$

\subsection{Laplace Transform in Discrete Time}

We may also express the equilibrium conditions in terms of the discrete Laplace transform:

$$
\hat{f}(\theta)=\sum_{z \geq 0} \mathrm{e}^{-\theta z} f(z)
$$

Hence

$$
\sum_{z \geq 0} \mathrm{e}^{-\theta z}=\frac{1}{1-\mathrm{e}^{-\theta}}
$$

and differentiating wrt to $\theta$ : 


$$
\sum_{z \geq 0} z \mathrm{e}^{-\theta z}=\frac{\mathrm{e}^{-\theta}}{\left[1-\mathrm{e}^{-\theta}\right]^{2}}
$$

For the consumption function $c=\gamma+r z$ :

$$
\begin{aligned}
& \hat{c}(\mu+r)=\frac{\gamma}{1-\mathrm{e}^{-(\mu+r)}}+\frac{r}{\left[1-\mathrm{e}^{-(\mu+r)}\right]^{2}} \\
& \hat{c}(\mu+\theta)=\frac{\gamma}{1-\mathrm{e}^{-(\mu+\theta)}}+\frac{r}{\left[1-\mathrm{e}^{-(\mu+\theta)}\right]^{2}}
\end{aligned}
$$

We suppose that labor is constant $b(z)=b$ for $m \leq z \leq n$ :

$$
\begin{aligned}
& \hat{b}(\mu+r)=b \frac{\mathrm{e}^{-(\mu+r) m}-\mathrm{e}^{-(\mu+r) n}}{1-\mathrm{e}^{-(\mu+r)}} \\
& \hat{b}(\mu+\theta)=b \frac{\mathrm{e}^{-(\mu+\theta) m}-\mathrm{e}^{-(\mu+\theta) n}}{1-\mathrm{e}^{-(\mu+\theta)}}
\end{aligned}
$$

The equilibrium conditions become:

$$
\hat{c}(\mu+r)=w \hat{b}(\mu+r)
$$

and

$$
\hat{c}(\mu+\theta)=\left[f-k\left(1-\mathrm{e}^{-\theta}\right)\right] \hat{b}(\mu+\theta)
$$

We suppose that labor is constant $b(z)=b$ for $m \leq z \leq n$ :

$$
\begin{aligned}
& \hat{b}(\mu+r)=b \frac{\mathrm{e}^{-(\mu+\theta) m}-\mathrm{e}^{-(\mu+\theta) n}}{1-\mathrm{e}^{-(\mu+\theta)}} \\
& \hat{b}(\mu+\theta)=b \frac{\mathrm{e}^{-(\mu+\theta) m}-\mathrm{e}^{-(\mu+\theta) n}}{1-\mathrm{e}^{-(\mu+\theta)}}
\end{aligned}
$$

so that

$$
\begin{gathered}
\gamma+\frac{r}{1-\mathrm{e}^{-(\mu+\theta)}}=w b\left[\mathrm{e}^{-(\mu+\theta) m}-\mathrm{e}^{-(\mu+\theta) n}\right] \\
\gamma+\frac{r}{1-\mathrm{e}^{-(\mu+\theta)}}=\left[f-k\left(1-\mathrm{e}^{-\theta}\right)\right] b\left[\mathrm{e}^{-(\mu+\theta) m}-\mathrm{e}^{-(\mu+\theta) n}\right]
\end{gathered}
$$

Hence

$$
b=\frac{1}{w} \frac{\gamma+\frac{r}{1-\mathrm{e}^{-(\mu+r)}}}{\mathrm{e}^{-(\mu+r) m}-\mathrm{e}^{-(\mu+r) n}}=\frac{1}{f-k\left(1-\mathrm{e}^{-\theta}\right)} \frac{\gamma+\frac{r}{1-\mathrm{e}^{-(\mu+\theta)}}}{\mathrm{e}^{-(\mu+\theta) m}-\mathrm{e}^{-(\mu+\theta) n}}
$$

Hence

$$
\frac{1}{\beta k^{\alpha}} \frac{\gamma+\frac{r}{\mathrm{e}^{-(\mu+r) m}-\mathrm{e}^{-(\mu+\theta)}}}{1(\mu+r) n}=\frac{1}{f-k\left(1-\mathrm{e}^{-\theta}\right)} \frac{\gamma+\frac{r}{1-\mathrm{e}^{-(\mu+\theta)}}}{\mathrm{e}^{-(\mu+\theta) m}-\mathrm{e}^{-(\mu+\theta) n}}
$$

or 


$$
\frac{\gamma+\frac{r}{1-\mathrm{e}^{-(\mu+r)}}}{\gamma+\frac{r}{1-\mathrm{e}^{-(\mu+\theta)}}}=\frac{\beta k^{\alpha}}{f-k\left(1-\mathrm{e}^{-\theta}\right)} \frac{\mathrm{e}^{-(\mu+r) m}-\mathrm{e}^{-(\mu+r) n}}{\mathrm{e}^{-(\mu+\theta) m}-\mathrm{e}^{-(\mu+\theta) n}}
$$

This condition suggests that $r=\theta$ is almost a solution of 14, if we make the approximation $1-\mathrm{e}^{-\theta} \simeq \theta$. The complete solutions illustrate this in Table 1 .

Blanks in the table indicate the absence of a significant growth rate. It is evident that the higher the interest rate, the higher the production per capita, and thus the higher the consumption that can be afforded, or the higher the population growth rate. (Note that the absence of a growth rate does not mean that it is zero.)

Samuelson's "golden rule" holds only approximately in the table above for discrete time. In the following section we shall see that it holds precisely in continuous time.

Table 1. Interest and growth rates, discrete time.

\begin{tabular}{|c|c|c|c|c|c|}
\hline $\begin{array}{c}\text { Interest Rate } \\
r\end{array}$ & $\begin{array}{c}\text { Growth rate } \\
\theta_{1}\end{array}$ & $\theta_{2}$ & $\theta_{3}$ & Wage $w$ & $\begin{array}{l}\text { Percapita } \\
\text { capital } k\end{array}$ \\
\hline $0.005 \%$ & $0.005 \%$ & & & 4.05 & 346.91 \\
\hline $0.010 \%$ & $0.010 \%$ & & & 3.01 & 128.88 \\
\hline $0.015 \%$ & $0.015 \%$ & & & 2.53 & 72.21 \\
\hline $0.020 \%$ & $0.020 \%$ & & & 2.23 & 47.88 \\
\hline $0.025 \%$ & $0.026 \%$ & & & 2.03 & 34.81 \\
\hline $0.030 \%$ & $0.032 \%$ & & & 1.88 & 26.83 \\
\hline $0.035 \%$ & $0.087 \%$ & & & 1.76 & 21.52 \\
\hline $0.040 \%$ & $0.021 \%$ & $0.038 \%$ & $0.118 \%$ & 1.66 & 17.79 \\
\hline $0.045 \%$ & $0.014 \%$ & $0.043 \%$ & $0.144 \%$ & 1.58 & 15.03 \\
\hline $0.050 \%$ & $0.009 \%$ & $0.049 \%$ & $0.169 \%$ & 1.51 & 12.93 \\
\hline $0.055 \%$ & $0.006 \%$ & $0.054 \%$ & $0.192 \%$ & 1.45 & 11.29 \\
\hline $0.060 \%$ & $0.004 \%$ & $0.059 \%$ & $0.215 \%$ & 1.40 & 9.97 \\
\hline $0.065 \%$ & $0.002 \%$ & $0.064 \%$ & $0.238 \%$ & 1.35 & 8.89 \\
\hline $0.070 \%$ & $0.069 \%$ & $0.261 \%$ & & 1.31 & 8.00 \\
\hline $0.075 \%$ & $0.074 \%$ & $0.284 \%$ & & 1.27 & 7.25 \\
\hline $0.080 \%$ & $0.079 \%$ & $0.308 \%$ & & 1.23 & 6.61 \\
\hline $0.085 \%$ & $0.084 \%$ & $0.331 \%$ & & 1.20 & 6.06 \\
\hline $0.090 \%$ & $0.089 \%$ & $0.355 \%$ & & 1.17 & 5.58 \\
\hline $0.095 \%$ & $0.094 \%$ & $0.380 \%$ & & 1.15 & 5.17 \\
\hline $0.100 \%$ & $0.099 \%$ & $0.405 \%$ & & 1.12 & 4.80 \\
\hline
\end{tabular}




\subsection{Continuous Time}

For analysis in continuous time, the variables are similar (but the outcomes are rather different). Assume the population at time suffers a mortality rate $\mu$, and that those born at time $s$ surviving to time $t$ is $\left[1-\mathrm{e}^{-(\mu+\theta)}\right] \mathrm{e}^{\theta s} \mathrm{e}^{-\mu(t-s)}$. Hence the total population at time $t$ is

$$
\begin{aligned}
L(t) & =\int_{s \leq t}(\mu+\theta) \mathrm{e}^{\theta s} \mathrm{e}^{-\mu(t-s)} \mathrm{d} s \\
& =(\mu+\theta) \mathrm{e}^{\theta t} \int_{z \geq 0} \mathrm{e}^{-(\mu+\theta) z} \mathrm{~d} z \\
& =\mathrm{e}^{\theta t} .
\end{aligned}
$$

which is of exponential growth. The total consumption function at time $t$ is then, using $z=t-s$ as the attained age of an individual:

$$
C(t)=\int_{s \leq t}(\mu+\theta) \mathrm{e}^{\theta s} \mathrm{e}^{-\mu(t-s)} c(t-s) \mathrm{d} s=(\mu+\theta) \mathrm{e}^{\theta t} \int_{0}^{\infty} \mathrm{e}^{-(\mu+r) z} c(z) \mathrm{d} z .
$$

For any generation $s$, the per capita value of future consumption at a constant interest rate $r$ is

$$
c=\int_{s \leq t}(\mu+\theta) \mathrm{e}^{-\mu(t-s)} c(t-s) \mathrm{e}^{-r(t-s)} \mathrm{d} t=\int_{0}^{\infty} \mathrm{e}^{-(\mu+r) z} c(z) \mathrm{d} z
$$

The value of per capita future income $b(z)$ at a unit wage rate for an individual aged $z$ is similarly

$$
b=\int_{s \leq t}(\mu+\theta) \mathrm{e}^{-\mu(t-s)} b(t-s) \mathrm{e}^{-r(t-s)} \mathrm{d} t=\int_{0}^{\infty} \mathrm{e}^{-(\mu+r) z} b(z) \mathrm{d} z .
$$

Note that the economy is steady state, as consumption and income are the same for all individuals over time, and interest rates and wage rates are constant (in real terms), notwithstanding constant exponential growth of the population and the economy as a whole.

Thus

$$
F=B f(k)=(\mu+\theta) f(k) \mathrm{e}^{\theta t} \int_{0}^{\infty} \mathrm{e}^{-(\mu+\theta) z} b(z) \mathrm{d} z
$$

Thus the conditions for stationarity of an equilibrium are the equality of the value of lifetime future income (at a wage rate $w$ ) and of consumption for any generation:

$$
\int_{0}^{\infty} \mathrm{e}^{-(\mu+r) z} c(z) \mathrm{d} z=w \int_{0}^{\infty} \mathrm{e}^{-(\mu+r) z} b(z) \mathrm{d} z
$$

and at any time the growth of capital through production not consumed in as 14

$$
\int_{0}^{\infty} \mathrm{e}^{-(\mu+\theta) z} c(z) \mathrm{d} z=(f-k \theta) \int_{0}^{\infty} \mathrm{e}^{-(\mu+\theta) z} b(z) \mathrm{d} z
$$

Given a framework for consumption and production of the economy these relations link $r$ and $\theta$. It is clear that $r=\theta$ is a solution to these relations 
(Samuelson's "golden rule"). But as the examples show below, they are by no means unique, even in steady state economies.

\section{Laplace Transform in Continuous Time}

The equilibrium conditions above may be expressed succinctly in terms of the Laplace transform, defined generally as:

$$
\hat{f}(\theta)=\int_{z \geq 0} \mathrm{e}^{-\theta z} f(z) \mathrm{d} z
$$

whence

$$
\int_{z \geq 0} \mathrm{e}^{-\theta z} f^{\prime}(z) \mathrm{d} z=\theta \int_{z \geq 0} \mathrm{e}^{-\theta z} f(z) \mathrm{d} z
$$

so that if $f(z)=z^{n}$

$$
\int_{z \geq 0} \mathrm{e}^{-\theta z} f(z) \mathrm{d} z=\frac{n}{\theta} \int_{z \geq 0} \mathrm{e}^{-\theta z} z^{n-1} \mathrm{~d} z
$$

and by induction

$$
\hat{z}^{n}=\frac{n !}{\theta^{n+1}} .
$$

The equilibrium conditions then become:

$$
\begin{aligned}
\hat{c}(\mu+r) & =\frac{\gamma}{\mu+r}+\frac{r}{(\mu+r)^{2}} \\
& =w \hat{b}(\mu+r) \\
& =\beta k^{\alpha}
\end{aligned}
$$

and

$$
\begin{aligned}
\hat{c}(\mu+\theta) & =\frac{\gamma}{\mu+\theta}+\frac{r}{(\mu+\theta)^{2}} \\
& =(f-k \theta) \hat{b}(\mu+\theta)
\end{aligned}
$$

where

$$
w=f-k f^{\prime}(k)=\beta k^{\alpha} .
$$

Eliminating $b, 22$ and 25 and become:

$$
\begin{aligned}
b & =\left[\gamma+\frac{r}{1+\theta}\right] \frac{1}{\beta k^{\alpha}} \frac{1}{\mathrm{e}^{-(\mu+r) m}-\mathrm{e}^{-(\mu+r) n}} \\
& =\left[\gamma+\frac{r}{1+r}\right] \frac{1}{f-k \theta} \frac{1}{\mathrm{e}^{-(1+\theta) m}-\mathrm{e}^{-(1+\theta) n}}
\end{aligned}
$$

or

$$
\frac{\gamma+\frac{r}{1+r}}{\gamma+\frac{r}{1+\theta}}=\frac{\beta k^{\alpha}}{f-k \theta} \frac{\mathrm{e}^{-(1+\theta) m}-\mathrm{e}^{-(1+\theta) n}}{\mathrm{e}^{-(\mu+r) m}-\mathrm{e}^{-(\mu+r) n}}
$$

which is exactly satisfied for $\theta=r$. 


\section{Conclusions}

The central result of this paper is contained in Proposition 16, which sets out the conditions for demographic efficiency as a particular form of budget constraint for all generations. The examples provided in [24] suggest that these conditions may be sufficient to specify completely the long run behavior of an economy.

The generational budget constraints may thus be seen as linkages, under price equilibrium, between the development of interest rates; technological progress, as shown by the trend to higher order commodities covered by the production function; and population movement. Whereas allocative efficiency provides qualitative conditions for these linkages, demographic efficiency provides quantitative ones.

This result has ramifications for retirement incomes policy, which seeks to achieve objectives for capital accumulation of consumers. These policies are based on transfers of wealth by government between different generations, via taxation, via subsidies granted to the needy, or even via monetary policy. They suffer the risk that population movements, and/or the course of interest rates, will in the long term compromise the objectives of those policies. And they also suggest that population policies, such as birth control, paid parental leave, forced sterilization, may have unpredictable consequences for the economy.

These conclusions have been reached under very restrictive conditions. The framework of this paper does not allow for altruism on the part of consumers in planning future consumption [25]. Nor does it allow for uncertainty in production, which would lead to uncertainty in consumption plans. It remains to be seen whether these features result in serious modification of the results presented in this paper.

\section{Conflicts of Interest}

The author declares no conflicts of interest regarding the publication of this paper.

\section{References}

[1] Samuelson, P.A. (1975) The Optimum Growth Rate for Population. International Economic Review, 16, 531-538. https://doi.org/10.2307/2525993

[2] Samuelson, P.A. (1975) Optimum Social Security in a Life-Cycle Growth Model. International Economic Review, 16, 539-544. https://doi.org/10.2307/2525994

[3] Samuelson, P.A. (1976) The Optimum Growth Rate for Population: Agreement and Evaluations. International Economic Review, 17, 516-525. https://doi.org/10.2307/2525721

[4] Cass, D. (1972) On Capital over Accumulation in the Aggregative, Neoclassical Model of Economic Growth: A Complete Characterisation. Journal of Economic Theory, 4, 200-223. https://doi.org/10.1016/0022-0531(72)90149-4

[5] Balasko, Y., Cass, D. and Shell, K. (1980) The Overlapping Generations Model I: The Case of Pure Exchange without Money. Journal of Economic Theory, 23, 307-322. https://doi.org/10.1016/0022-0531(80)90014-9 
[6] Wilson, C.A. (1981) Equilibrium in Dynamic Models with an Infinity of Agents. Journal of Economic Theory, 24, 95-111. https://doi.org/10.1016/0022-0531(81)90066-1

[7] Peleg, B. and Yaari, M.E. (1970) Markets with Countably Many Commodities. International Economic Review, 11, 369-377. https://doi.org/10.2307/2525317

[8] Richard, S.F. and Srivastava, S. (1988) Equilibrium in Economies with Infinitely Many Consumers and Infinitely Many Commodities. Journal of Mathematical Economics, 17, 9-21. https://doi.org/10.1016/0304-4068(88)90023-7

[9] Bewley, T.F. (1972) Existence of Equilibria in Economies with Infinitely Many Commodities. Journal of Economic Theory, 4, 514-540. https://doi.org/10.1016/0022-0531(72)90136-6

[10] Mas-Collell, A. (1975) A Model of Equilibrium with Differentiated Commodities. Journal of Mathematical Economics, 2, 263-296. https://doi.org/10.1016/0304-4068(75)90028-2

[11] Ostroy, J.M. (1984) On the Existence of Walrasian Equilibria in Large Square Economies. Journal of Mathematical Economics, 13, 143-164. https://doi.org/10.1016/0304-4068(84)90014-4

[12] Pazner, E.A. and Razin, A. (1980) Competitive Efficiency in an Overlapping Generation Model with Endogenous Population. Journal of Public Economics, 13, 249-258. https://doi.org/10.1016/0047-2727(80)90015-8

[13] Nerlove, M., Razin, A. and Sadka, E. (1987) Household and Economy. Academic Press, Amsterdam.

[14] D'Albis, H. (2007) Demographic Structure and Capital Accumulation. Journal of Economic Theory, 132, 411-434. https://doi.org/10.1016/j.jet.2005.10.001

[15] Lau, S.-H.P. (2009) Demographic Structure and Capital Accumulation: A Quantitative Assessment. Journal of Economic Dynamics and Control, 33, 554-567. https://doi.org/10.1016/j.jedc.2008.08.004

[16] Ostroy, J.M. (1980) The No-Surplus Condition as a Characterization of Perfectly Competitive Equilibrium. Journal of Economic Theory, 22, 183-207. https://doi.org/10.1016/0022-0531(80)90039-3

[17] Diamond, P.A. (1965) National Debt in a Neoclassical Growth Model. American Economic Review, 55, 1126-1150.

[18] Bowers, N.L., Gerber, H.U., Hickman, J.C., Jones, D.A. and Nesbitt, C.J. (1986) Actuarial Mathematics. Society of Actuaries, Schaumburg.

[19] Kihlstrom, R.E. and Mirman, L.J. (1974) Risk Aversion with Many Commodities. Journal of Economic Theory, 8, 361-388. https://doi.org/10.1016/0022-0531(74)90091-X

[20] M E Yaari. (1965) Uncertain Lifetime, Life Insurance and the Theory of the Consumer. The Review of Economics Studies, 32, 137-150. https://doi.org/10.2307/2296058

[21] Robertson, A.P. and Robertson, W. (1973) Topological Vector Spaces. Cambridge University Press, Cambridge.

[22] Dunford, N. and Schwartz, J.T. (1970) Linear Operators: Part I. Interscience, Hoboken.

[23] Conway, J.B. (1967) The Strict Topology and Compactness in the Space of Measures II. Transactions of the American Mathematical Society, 126, 474-486. https://doi.org/10.1090/S0002-9947-1967-0206685-2 
[24] Leung, A.P. (2001) Interest Rates, Population and Technology. North American Actuarial Journal, 5, 43-52.

[25] Raut, L.K. (2006) Two-Sided Altruism, Lindahl Equilibrium, and Pareto Optimality in Overlapping Generations Models. Economic Theory, 27, 729-736. https://doi.org/10.1007/s00199-004-0562-9

[26] Abramovich, Y.A., Aliprantis, C.D. and Zame, W.R. (1995) A Representation Theorem for Riesz Spaces and Its Applications to Economics. Economic Theory, 5, 527-535. https://doi.org/10.1007/BF01212332 


\section{Appendix: Proofs of Results in Section 6}

Lemma 11

Proof. For all $\mathbf{v} \in l_{1}(\mathbb{Z}), \quad \mathbf{c} \in l_{\infty}\left(\mathbb{Z}^{2}\right)$ we have

$$
|\langle\mathbf{v}, \pi \mathbf{c}\rangle| \equiv\left|\sum_{t} \mathbf{v}(t) \cdot \pi \mathbf{c}(t) L(t)\right| \leq \sum_{s, t}|\mathbf{v} \cdot \mathbf{c}| \ell<\|\mathbf{v}\|_{1}\|\mathbf{c}\|_{\infty}<\infty .
$$

Hence $\pi \mathbf{c}$ defines a continuous linear functional on $l_{1}(\mathbb{Z})$ and $\pi \mathbf{c} \in l_{\infty}(\mathbb{Z})$. If $\mathbf{c} \in l_{\infty}\left(\mathbb{Z}^{2}\right)$ is taken to be of the form $\mathbf{c}(s, t)=\mathbf{c}(t) \in l_{\infty}(\mathbb{Z})$, then $\pi \mathbf{c}=\mathbf{c}$, so that the map $\pi$ is surjective. Hence by the open mapping principle [[22], §II.2.1], the map is open.

\section{Proposition 12}

Proof. This is essentially Theorem 1 of [8], but the proof given here relies on establishing an interior point in the set of improving variations, which result is needed in Section 6.2.

The proof is similar to that of the second welfare theorem for finite economies, but requires the application of separating hyperplane theorems for infinite dimensional spaces. Using the properties of the utility function $u(\mathbf{c})$ it is not difficult to show that $\mathcal{P}$ and hence $\pi \mathcal{P}$ is convex. We show that $\pi \mathcal{P}$ has an interior point in the strong topology of $l_{\infty}(\mathbb{Z})$, analogous to the existence of interior points in the positive cone of $l_{\infty}(\mathbb{Z})[26]$.

Since $\mathbf{c}^{*}$ is bounded, there exists an allocation $\overline{\mathbf{c}} \geq \mathbf{c}^{*}$ and a constant $m>0$ such that $u(\overline{\mathbf{c}})-u\left(\mathbf{c}^{*}\right) \geq m$ for all $(s, t)$, with $\overline{\mathbf{c}}$ being bounded both above and below. It is clear that $\overline{\mathbf{c}} \in \mathcal{P}$; we claim that $\pi \overline{\mathbf{c}}$ is an interior point of $\pi \mathcal{P}$.

Let $K>0$ be the assumed upper bound on $\left|u^{\prime}\right|$, and let $k>0$ be the curvature parameter relating to maximum curvature, ie. $-x^{\mathrm{T}} u^{\prime \prime} x \leq k\left|u^{\prime}\right||x|^{2}$. Using Taylor's theorem with remainder for a variation $\Delta \mathbf{c}$, we have for some $0<\theta<1$ :

$$
\begin{aligned}
|u(\overline{\mathbf{c}}+\Delta \mathbf{c})-u(\overline{\mathbf{c}})| & =\left|u^{\prime}(\overline{\mathbf{c}}) \cdot \Delta \mathbf{c}+\frac{1}{2} \Delta \mathbf{c}^{\mathrm{T}} u^{\prime \prime}(\overline{\mathbf{c}}+\theta \Delta \mathbf{c}) \Delta \mathbf{c}\right| \\
& \leq\left|u^{\prime}(\overline{\mathbf{c}}) \cdot \Delta \mathbf{c}\right|+\frac{1}{2} k\left|u^{\prime}(\overline{\mathbf{c}}+\theta \Delta \mathbf{c})\right||\Delta \mathbf{c}|^{2} \\
& \leq K \mid \Delta \mathbf{c}\left(1+\frac{1}{2} k|\Delta \mathbf{c}|\right) .
\end{aligned}
$$

Thus for any sufficiently small $q<1$, we can find $\varepsilon>0$ so that for all variations $\Delta \mathbf{c}$ to $\overline{\mathbf{c}}$ with $\|\Delta \mathbf{c}\|_{\infty} \leq \varepsilon$, we have

$$
|u(\overline{\mathbf{c}}+\Delta \mathbf{c})-u(\overline{\mathbf{c}})| \leq m q .
$$

Hence for any generation $s$, we have

$$
\begin{aligned}
U^{s}(\overline{\mathbf{c}}+\Delta \mathbf{c})-U^{s}\left(\mathbf{c}^{*}\right) & =\sum_{t}\left[u(\overline{\mathbf{c}}+\Delta \mathbf{c})-u\left(\mathbf{c}^{*}\right)\right] \ell \\
& \geq \sum_{t}\left[u(\overline{\mathbf{c}})-u\left(\mathbf{c}^{*}\right)\right] \ell-\sum_{t}|u(\overline{\mathbf{c}}+\Delta \mathbf{c})-u(\overline{\mathbf{c}})| \ell \\
& \geq(1-q) m>0 .
\end{aligned}
$$


This implies that there is a neighborhood of $\overline{\mathbf{c}}$ which is mapped by $\pi$ into $\pi \mathcal{P}$. Since $\pi$ is an open mapping under Lemma $11, \pi \overline{\mathbf{c}}$ is an interior point of $\pi \mathcal{P}$.

Since $\mathbf{c}^{*}$ is efficient, we must have $\pi \mathbf{c}^{*} \notin \pi \mathcal{P}$. Applying a separation theorem for convex sets with non-empty interior to the set $\pi \mathcal{P}$, [[22], §V.2.8], there exists $\mathbf{v} \in b a(\mathbb{Z})$ with the required property.

Lemma 14

Proof. This follows from the fact that $\mathbf{v}(t)$ supports the allocation $\mathbf{c}^{*}$ subject to each generation's budget constraint ${ }^{7}$ in Equation (2).

Proposition 15

Proof. This is essentially Theorem 2 of [8], which shows that the countably additive component of $\mathbf{v} \in b a(\mathbb{Z})$ is also a separating functional in $l_{1}(\mathbb{Z})$. It suffices to show that the preferences induced by the utility function $U^{s}[\mathbf{c}]$ are continuous in the Mackey topology $\tau\left(l_{\infty}, l_{1}\right)$, which is proved formally in [[9], Appendix II]. However, under the present conditions, a simpler proof is possible.

Let $\left\{\mathbf{c}_{n} \in l_{\infty}\left(\mathbb{Z}^{2}\right)\right\}$ be a sequence of allocations which converges to $\mathbf{c}$ in the Mackey topology. We need to show that $U^{s}\left[\mathbf{c}_{n}\right] \rightarrow U^{s}[\mathbf{c}]$.

Using the characterization (1), we see that the sequence $\left\{\left|\mathbf{c}_{n}-\mathbf{c}\right| \in l_{\infty}\left(\mathbb{Z}^{2}\right)\right\}$ converges to zero in the Mackey topology, and therefore also in the weak topology $^{8} \sigma\left(l_{\infty}, l_{1}\right)$.

Using the inequality (27), we have

$$
\left|u\left(\mathbf{c}_{n}\right)-u(\mathbf{c})\right| \leq K\left|\Delta \mathbf{c}_{n}\right|\left[1+\frac{k}{2}\left|\Delta \mathbf{c}_{n}\right|\right]
$$

where $\Delta \mathbf{c}_{n}=\mathbf{c}_{n}-\mathbf{c}$. As $\left\{\Delta \mathbf{c}_{n}\right\}$ is weakly bounded, it must also be strongly bounded, so that there exists a constant $M>0$ so that

$$
\left|u\left(\mathbf{c}_{n}\right)-u(\mathbf{c})\right| \leq M\left|\Delta \mathbf{c}_{n}\right|
$$

and thus

$$
\left|U^{s}\left[\mathbf{c}_{n}\right]-U^{s}[\mathbf{c}]\right| \leq M \sum_{t} \ell(s, t)\left|\Delta \mathbf{c}_{n}\right| \rightarrow 0 .
$$

The remainder of the proof follow from Lemma 14.

Proposition 16

Proof. Let $\pi$ be the average consumption map of Lemma 11, and let $\mathcal{P}\left[\mathbf{c}^{*}\right]$ be the set of improving allocations for $\mathbf{c}^{*}$ as used in the proof of Proposition 12. Under the given conditions it has been shown that

- $\pi$ is a continuous open map (Lemma 11);

- $\pi \mathcal{P}$ has an interior point in the strong topology of $l_{\infty}(\mathbb{Z})$ (Proposition 12); and

- the discount functional $\mathbf{v} \in l_{1}\left(\mathbb{Z}^{2}\right)$ separating $\pi \mathbf{c}^{*}$ and $\pi \mathcal{P}$ is unique up

${ }^{7}$ It may be noted that uniqueness of the supporting price requires only one generation to have smooth preferences in the form of $U^{s}[\mathbf{c}]$.

${ }^{8}$ As stated in [23], this is the only consequence of Mackey convergence that is needed. 
to a multiplicative constant (Proposition 15 and Lemma 14).

From the concavity properties of the production function it is easy to show that $\mathcal{Q}$ and thus $\pi \mathcal{Q}$ is a convex set. Since $\mathbf{c}^{*}$ is demographically efficient, we have $\mathbf{c}^{*} \in \mathcal{Q}, \pi \mathbf{c}^{*} \notin \pi \mathcal{P}$ and $\pi \mathcal{P} \cap \pi \mathcal{Q}=\varnothing$. Applying a separation theorem for disjoint convex sets, of which the set $\pi \mathcal{P}$ has non-empty interior, [[22], $\S$ V.2.8], there exists a functional $\mathbf{z}(t) \in b a(\mathbb{Z})$ separating $\pi \mathcal{P}$ and $\pi \mathcal{Q}$, that is there exists a number $q$ such that

$$
\langle\mathbf{z}, \pi \mathcal{Q}\rangle \leq q \leq\langle\mathbf{z}, \pi \mathcal{P}\rangle .
$$

Using the inequality $\left\langle\mathbf{z}, \pi \mathbf{c}^{*}\right\rangle \leq\langle\mathbf{z}, \pi \mathcal{P}\rangle$, which holds to any desired accuracy, we can take $q=\left\langle\mathbf{z}, \pi \mathbf{c}^{*}\right\rangle$.

Consider the Yoshida-Hewitt decomposition of $\mathbf{z}=\mathbf{z}_{c}+\mathbf{z}_{p}$ into countably additive and purely finitely additive components. From Proposition $15, \mathbf{z}_{c}$ also separates $\pi \mathbf{c}^{*}$ and $\pi \mathcal{P}$. But the discount functional $\mathbf{v}$ separating $\pi \mathbf{c}^{*}$ and $\pi \mathcal{P}$ is unique, so we can take $\mathbf{z}_{c}=\mathbf{v}$.

Now consider the separation property $\langle\mathbf{z}, \pi \mathcal{Q}\rangle \leq\left\langle\mathbf{z}, \pi \mathbf{c}^{*}\right\rangle$. For any $n \in l_{\infty}(\mathbb{Z})$ with finite support, the resulting variations $\Delta B$ and thus $\Delta \mathbf{F}$ also have finite support. This follows from Equation (4) and the assumption that each generation has finite lifetime. As the purely additive component $\mathbf{z}_{p}$ is zero on finite intervals, this means that for all birth variations $n(s) \in l_{\infty}(\mathbb{Z})$ with finite support the separation property 30 becomes:

$$
\sum_{t} \mathbf{v}(t) \cdot\left[\Delta \mathbf{F}-\sum_{s} \mathbf{c}^{*} n \ell\right] \leq 0 .
$$

Using Taylor's theorem with remainder, we have

$$
\begin{aligned}
\Delta \mathbf{F} & =\mathbf{F}(\mathbf{K}, B+\Delta B)-\mathbf{F}(\mathbf{K}, B) \\
& =\mathbf{F}_{B} \Delta B+\frac{1}{2} \Delta B^{2} \mathbf{F}_{B B}(B+\theta \Delta B) \text { for some } 0 \leq \theta \leq 1 .
\end{aligned}
$$

Thus we have

$$
\mathbf{v}(t) \cdot \Delta \mathbf{F} \geq \mathbf{v}(t) \cdot \mathbf{F}_{B} \Delta B-\frac{1}{2} M \Delta B^{2}|\mathbf{v}(t)|,
$$

and for any $n \in l_{\infty}(\mathbb{Z})$ with finite support we have

$$
\sum_{s, t} \mathbf{v}(t) \cdot\left[b \mathbf{F}_{B}-\mathbf{c}^{*}\right] n(s) \ell \leq \frac{1}{2} M \sum_{t}|\mathbf{v}(t)|\left[\sum_{s} b n \ell\right]^{2} .
$$

We now apply a continuity argument by taking successively "smaller" birth functions $n \in l_{\infty}(\mathbb{Z})$. For a given $n \in l_{\infty}(\mathbb{Z})$, consider the functions $\varepsilon n \in l_{\infty}(\mathbb{Z})$ for arbitrary $\varepsilon \in \mathbb{Z}$. The above inequality implies that

$$
\begin{aligned}
& \varepsilon \sum_{s, t} \mathbf{v}(t) \cdot\left[b \mathbf{F}_{B}-\mathbf{c}^{*}\right] n(s) \ell \\
& \leq \frac{1}{2} M \varepsilon^{2} \sum_{t} \mid \mathbf{v}(t)\left[\left[\sum_{s} b n \ell\right]^{2}\right. \\
& \leq \frac{1}{2} M \varepsilon^{2}\|\mathbf{v}(t)\|\left\|\sum_{s} b n \ell\right\|_{\infty}^{2} .
\end{aligned}
$$


In the limit as $\varepsilon \rightarrow 0$ we must have $\sum \mathbf{v}(t) \cdot\left[b \mathbf{F}_{B}-\mathbf{c}^{*}\right] n(s) \ell \leq 0$. Since this holds for arbitrary $n \in l_{\infty}(\mathbb{Z})$, the conclusion follows. 